Article

\title{
Synthesis and Anti-Human Immunodeficiency Virus Type 1 Activity of (E)- $N$-Phenylstyryl- $N$-alkylacetamide Derivatives
}

Pi Cheng ${ }^{1, *}$, Ji-Jun Chen ${ }^{2}$, Ning Huang ${ }^{3}$, Rui-Rui Wang ${ }^{3}$, Yong-Tang Zheng ${ }^{3}$ and Yi-Zeng Liang ${ }^{1}$

1 School of Chemistry and Chemical Engineering, Central South University, Changsha 410083, China

2 State Key Laboratory of Phytochemistry and Plant Resources in West China, Kunming Institute of Botany, the Chinese Academy of Sciences, Kunming 650204, China

3 Laboratory of Molecular Immunopharmacology, Key Laboratory of Animal Models and Human Diseases Mechanisms, Kunming Institute of Zoology, the Chinese Academy of Sciences, Kunming 650223, China

* Author to whom correspondence should be addressed; E-mail: picheng55@126.com; Tel.: +86-731-8836376.

Received: 1 July 2009; in revised form: 30 July 2009 / Accepted: 26 August 2009 / Published: 26 August 2009

\begin{abstract}
A series of (E)- $N$-phenylstyryl- $N$-alkylacetamides, 5, were synthesized by direct reduction-acetylation of $\beta$-arylnitroolefins, followed by $N$-alkylation. The title compounds were characterized by ${ }^{1} \mathrm{H}-\mathrm{NMR}$, EIMS and IR analysis. All the synthesized compounds were assayed as HIV-1 non-nucleoside reverse transcriptase inhibitors. A SAR study revealed that when group $\mathrm{R}^{1}$ in $\mathbf{5}$ was ortho-substituted, the resulting compounds showed better inhibitory activities against HIV-1 RT. Among the tested compounds, $\mathbf{5 i}\left(\mathrm{R}^{1}=2-\mathrm{Br}\right.$, $\mathrm{R}^{2}=3,5$-difluorobenzyl) exhibited the highest enzyme activity, with a $88.89 \%$ inhibitory ratio against HIV-1 reverse transcriptase at the tested concentration. Further cell-based anti-HIV-1 assays showed that compound $\mathbf{5 i}$ exhibited a SI value of 29 with an $\mathrm{EC}_{50}$ value of $4 \mu \mathrm{M}$ in C8166 cells.
\end{abstract}

Keywords: $(E)-N$-phenylstyryl- $N$-alkylacetamides; synthesis; reverse transcriptase; antiHIV-1 activity 


\section{Introduction}

Acquired immunodeficiency syndrome (AIDS), a disease resulting from infection with Human Immunodeficiency Virus type 1 (HIV-1), is one of the world's most serious health problems. Three essential enzymes are encoded by the HIV-1 pol gene: (1) HIV-1 reverse transcriptase (RT), (2) integrase (IN), and (3) protease (PR). These three enzymes compose the most important and validated targets for developing novel antiretroviral agents [1-4]. Among RT inhibitors, non-nucleoside reverse transcriptase inhibitors (NNRTIs) remain a high priority for medical research because of their structural diversity [5-9].

Structure simplification of natural product has provided us an efficient way to find new and less toxic anti-HIV-1 lead compounds [10]. In a previous paper [11], we reported that (E)- $N$-phenylstyryl acetamides (Scheme 1), which contain the basic structural skeleton of hamigeroxalamic acid, could exhibit HIV-1 RT and showed for the first time moderate anti-HIV-1 activities in cell culture. Fundamental structure-activity relationships (SAR) were revealed and it was proposed that when group $\mathrm{R}^{1}$ was an ortho-substituent, the resulting compounds showed better inhibitory activities against HIV-1 RT. The ortho-substituted analogues thus represent a new template for further structure modification with a view to finding more active anti-HIV-1 analogues.

Scheme 1. Structure of hamigeroxalamic acid, $(E)-N$-phenylstyryl acetamide and $(E)-N$ phenylstyryl- $N$-alkylacetamide.

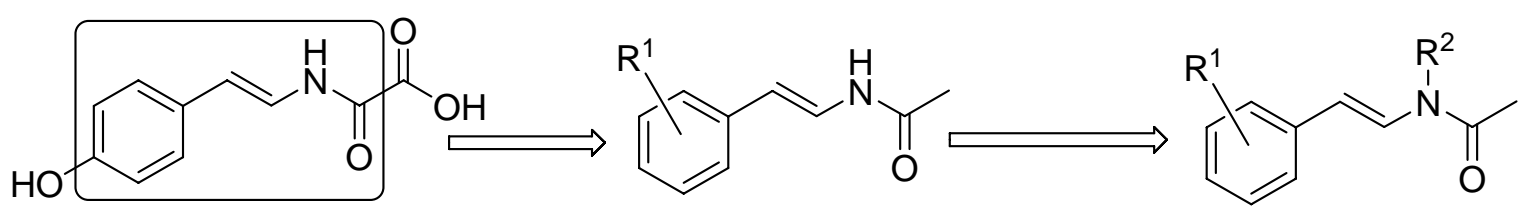

hamigeroxalamic acid

(E)-N-phenylstyryl acetamide

(E)-N-phenylstyryl- $N$-alkylacetamide

In this paper, a series of (E)- $N$-phenylstyryl- $N$-alkylacetamides (Scheme 1 ) were synthesized and evaluated in vitro as NNRTIs of HIV-1. Besides, syncytium reduction and cytotoxicity activities of the title compounds in cell based assay are provided. Detailed synthetic procedures and the anti-HIV-1 activities of these $N$-phenylstyryl- $N$-alkylacetamides are reported.

\section{Results and Discussion}

\subsection{Chemistry}

The synthetic route to the $(E)-N$-phenylstyryl- $N$-alkylacetamides $\mathbf{5}$ are shown in Scheme 2 . First, (E)- $\beta$-nitroolefins 2 were produced by refluxing various benzaldehydes with ammonium acetate and nitromethane. Compounds 3 were synthesized through a one-pot reduction of compounds 2 using $\mathrm{Fe} / \mathrm{AcOH} / \mathrm{Ac}_{2} \mathrm{O}$. In this reaction, diacetyl compounds 4 were isolated as byproducts. These could also be converted into compounds $\mathbf{3}$ by treatment of potassium hydroxide. Treatment of compounds $\mathbf{3}$ with sodium hydride and various alkyl halides provided the title compounds $\mathbf{5}$ in moderate yields. Structures of groups $\mathrm{R}^{1}$ and $\mathrm{R}^{2}$ were given in Scheme $\mathbf{2}$, and $\mathrm{R}^{1}$ were mainly substituted in the orthoposition on the aromatic ring. 
Scheme 2. Synthetic route for (E)- $N$-phenylstyryl- $N$-alkylacetamides (5).<smiles>[R]c1cccc(C)c1</smiles>

1

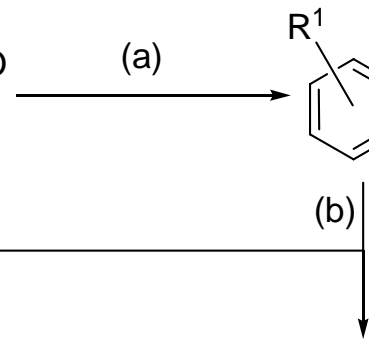<smiles>[R]c1ccccc1C=C[N+](=O)[O-]</smiles>

2<smiles>[Z1]c1ccccc1/C=C/N(C(C)=O)C(C)=O</smiles>

$\mathrm{R}^{1}=2-\mathrm{F}$

$2-\mathrm{Cl}$

$2-\mathrm{Br}$

2-methyl

2,4-dimethoxyl<smiles>[R1]c1ccccc1/C=C/NC(C)=O</smiles>

(c)

4

$$
\begin{aligned}
R^{2}= & \text { methyl } \\
& \text { ethyl } \\
& \text { allyl } \\
& \text { benzyl } \\
& \text { 2,6-difluorobenzyl } \\
& \text { 3,5-difluorobenzyl } \\
& \text { 2,6-dichlorobenzyl }
\end{aligned}
$$

Reagents and conditions: (a) $\mathrm{NH}_{4} \mathrm{OAc}, \mathrm{MeNO}_{2}$, reflux; (b)Fe/ $\mathrm{AcOH} / \mathrm{Ac}_{2} \mathrm{O}$, reflux, $\mathrm{N}_{2}$; (c) $\mathrm{KOH}$, $\mathrm{MeOH}$; (d) NaH, ${ }^{2} \mathrm{X}$, THF, $0{ }^{\circ} \mathrm{C}$.

A possible mechanism for the one-pot synthesis of (E)-N-phenylstyryl acetamides 3 by direct reduction-acetylation of $(E)-\beta$-phenyl-nitroolefins 2 is illustrated in Figure 2.

Figure 2. One-pot synthesis of $N$-phenylstyryl acetamides 3.

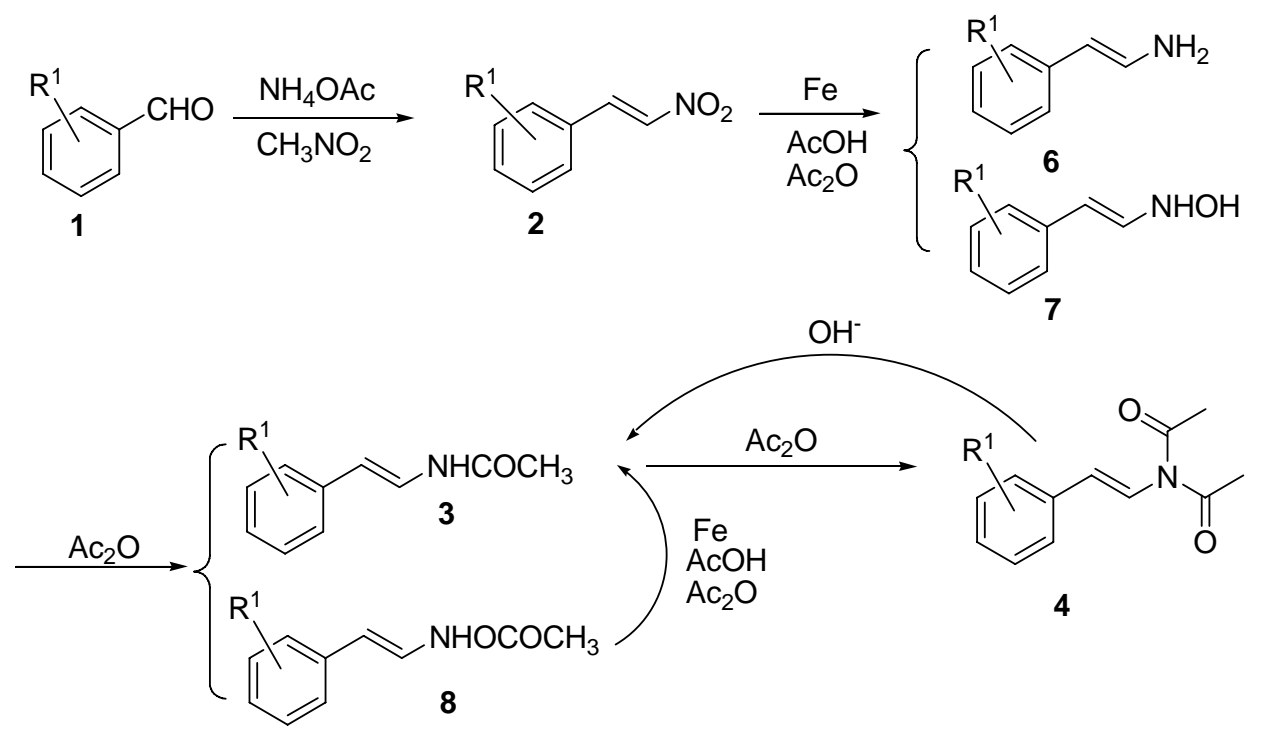

Compounds 2 would first be reduced to compounds 6 and/or 7. Compounds 7 would be trapped in the presence of acetic anhydride to form oxime acetates $\mathbf{8}$, as would compounds $\mathbf{6}$, to yield the desired (E)- $N$-phenylstyryl acetamides $\mathbf{3}$. Oxime acetates $\mathbf{8}$ should be in turn reduced to $\mathbf{3}$ under the reaction conditions. Compounds $\mathbf{3}$ might be further acetylated to the diacetyl compound $\mathbf{4}$, a reaction that could be reversed by treatment with methanolic potassium hydroxide. It was worth noting that the trans- 
conformation of the carbon-carbon double bonds was maintained in this reduction-acetylation reaction.

Table 1. Structures and anti-HIV-1 activities of (E)- $N$-phenylstyryl- $N$-alkylacetamides 5. ${ }^{\text {a }}$

\begin{tabular}{|c|c|c|c|c|c|c|c|}
\hline Entry & $\mathrm{R}^{1}$ & $\mathrm{R}^{2}$ & $\begin{array}{l}\mathrm{RT} \\
\text { inhibitio } \\
\mathrm{n}(\%)^{\mathrm{b}}\end{array}$ & $\begin{array}{l}\mathrm{IC}_{50}{ }^{\mathrm{c}} \\
(\mu \mathrm{M})\end{array}$ & $\begin{array}{l}\mathrm{EC}_{50}{ }^{\mathrm{d}} \\
(\mu \mathrm{M})\end{array}$ & $\begin{array}{l}\mathrm{CC}_{50}{ }^{\mathrm{e}} \\
(\mu \mathrm{M})\end{array}$ & $\begin{array}{l}\mathrm{SI}^{\mathrm{f}} \\
\left(\mathrm{CC}_{50} / \mathrm{EC}_{50}\right)\end{array}$ \\
\hline $5 \mathbf{a}$ & $3-\mathrm{Br}$ & methyl & 45.42 & ND & $128 \pm 20$ & $409 \pm 106$ & 3.2 \\
\hline $5 \mathbf{b}$ & $4-\mathrm{Br}$ & methyl & 16.59 & ND & ND & ND & ND \\
\hline $5 c$ & $2-\mathrm{Br}$ & methyl & 85.27 & $339 \pm 107$ & $60 \pm 10$ & $>789$ & $>13.2$ \\
\hline $5 d$ & $2-\mathrm{Br}$ & ethyl & 46.37 & ND & $48 \pm 8$ & $358 \pm 88$ & 7.5 \\
\hline $5 e$ & $2-\mathrm{Br}$ & allyl & 47.76 & ND & $75 \pm 13$ & $337 \pm 154$ & 4.5 \\
\hline $5 f$ & $2-\mathrm{Br}$ & acetyl & 60.55 & $320 \pm 22$ & $8 \pm 1$ & $85 \pm 17$ & 10.6 \\
\hline $5 \mathrm{~g}$ & $2-\mathrm{Br}$ & benzyl & 47.56 & ND & $9 \pm 2$ & $51 \pm 13$ & 5.7 \\
\hline $5 \mathbf{h}$ & $2-\mathrm{Br}$ & 2,6-difluoro-benzyl & 57.33 & $295 \pm 9$ & $29 \pm 4$ & $172 \pm 73$ & 5.9 \\
\hline $5 \mathbf{i}$ & $2-\mathrm{Br}$ & 3,5-difluoro-benzyl & 88.89 & $97 \pm 13$ & $4 \pm 0.5$ & $116 \pm 13$ & 29 \\
\hline $5 \mathbf{j}$ & $2-\mathrm{Br}$ & 2,6-dichloro-benzyl & 30.34 & ND & $49 \pm 10$ & $190 \pm 36$ & 3.9 \\
\hline $5 \mathbf{k}$ & $2-\mathrm{Cl}$ & methyl & 75.22 & $434 \pm 44$ & $96 \pm 20$ & $441 \pm 18$ & 4.6 \\
\hline 51 & $2-\mathrm{Cl}$ & ethyl & 59.10 & $521 \pm 11$ & $91 \pm 15$ & $424 \pm 22$ & 4.7 \\
\hline $5 \mathrm{~m}$ & $2-\mathrm{Cl}$ & allyl & 29.95 & ND & $118 \pm 28$ & $536 \pm 41$ & 4.5 \\
\hline $5 n$ & $2-\mathrm{F}$ & methyl & 61.69 & $477 \pm 107$ & $130 \pm 33$ & $664 \pm 79$ & 5.5 \\
\hline 50 & $2-\mathrm{F}$ & allyl & 38.51 & ND & $261 \pm 40$ & $538 \pm 138$ & 2.0 \\
\hline $5 p$ & 2-methyl & methyl & 79.80 & $487 \pm 27$ & $116 \pm 11$ & $609 \pm 177$ & 5.2 \\
\hline $5 \mathbf{q}$ & 2-methyl & allyl & 40.30 & ND & $273 \pm 43$ & $>930$ & $>3.4$ \\
\hline $5 r$ & 2,4-dimethoxy & methyl & 69.45 & $373 \pm 40$ & $32 \pm 7$ & $256 \pm 35$ & 8.0 \\
\hline $5 s$ & 2,4-dimethoxy & ethyl & 34.73 & ND & $19 \pm 3$ & $112 \pm 10$ & 5.9 \\
\hline $5 t$ & 2,4-dimethoxy & allyl & 36.62 & ND & $55 \pm 4$ & $290 \pm 79$ & 5.3 \\
\hline $\mathrm{AZT}^{\mathrm{g}}$ & & & $\mathrm{ND}^{\mathrm{i}}$ & ND & $1.08 \times 10^{-2}$ & $>509$ & $>47130$ \\
\hline $\mathrm{PFA}^{\mathrm{h}}$ & & & 96.46 & 5 & $\mathrm{ND}$ & ND & ND \\
\hline
\end{tabular}

${ }^{a}$ All data represent mean values for at least two separate experiments. ${ }^{b}$ All compounds were tested at 200 $\mu \mathrm{g} / \mathrm{mL} .{ }^{c}$ concentration that inhibit $50 \% \mathrm{HIV}-1 \mathrm{RT} .{ }^{\mathrm{e}} \mathrm{EC}_{50}=$ Effective concentration required to protect C8166 cells against the cytopathicity of $\mathrm{HIV}-1_{\text {III }}$ by $50 \% .{ }^{\mathrm{e}} \mathrm{CC}_{50}=$ Cytostatic concentration required to reduce $\mathrm{C} 8166$ cell proliferation by $50 \% .{ }^{\mathrm{f}}$ Selectivity index: $\mathrm{CC}_{50} / \mathrm{EC}_{50}$ ratio. ${ }^{\mathrm{g}} \mathrm{AZT}$ was used as positive control. ${ }^{\text {h}}$ PFA: Foscarnet was tested at $20 \mu \mathrm{g} / \mathrm{mL}$ as positive control. ${ }^{\mathrm{i}} \mathrm{ND}$ : not determined.

\subsection{Biological activity}

(E)- $N$-phenylstyryl- $N$-alkylacetamides 5 were assayed for their inhibitory ratio against HIV-1 RT at $200 \mu \mathrm{g} / \mathrm{mL}$. As outlined in Table 1, 5a and $\mathbf{5 b}$ showed significantly less enzymatic activity, compared with $\mathbf{5 c}$, which suggests that the presence of an ortho-substituent on the aromatic ring in the $N$-methyl derivatives appears to increase enzyme inhibition. This positive influence of a group in ortho position as to compounds $\mathbf{3}$ was already reported in our previous paper [11].

In some new developed NNRTIs such as the oxindole [12] and benzimidazole-2-one [13] derivatives, $\mathrm{NH}$ unit in the amide is a proton-donating group in the hydrogen bond formation process 
between the inhibitor and HIV-1 RT based on detailed docking studies, and $N$-alkyl analogues would suffer dramatic loss of activities. In our experiment, the SAR of compounds 5 against HIV-1 RT was similar to that of compounds 3 - the ortho substituted compounds possessed higher enzymatic activity. Furthermore, compounds 5 possessed higher inhibitory ratio against HIV-1 RT compared with their synthetic precursors $\mathbf{3}$. These suggested that compounds $\mathbf{5}$ and $\mathbf{3}$ probably have similar mechanism of action towards HIV-1 RT, and the NH unit in compounds 3 was not crucial to potent anti-HIV-1 RT activity.

The basic SAR prompted us to synthesize a series of $\mathrm{N}$-alkyl analogues $\mathbf{5 d}-\mathbf{5 t}$ with orthosubstituents $\mathrm{R}^{1}$ (Table 1). Compound $\mathbf{5 i}\left(\mathrm{R}^{1}=2-\mathrm{Br}, \mathrm{R}^{2}=3\right.$,5-difluorobenzyl) exhibited the highest enzyme activity. with a $88.89 \%$ inhibitory ratio against HIV-1 RT at the tested concentration, which increased nearly one fold from the precursor compound $\mathbf{3}\left(\mathrm{R}^{1}=2-\mathrm{Br}, \mathrm{R}^{2}=\mathrm{H}\right)$. Compounds $\mathbf{5}$ were then tested for their syncytium reduction activities $\left(\mathrm{EC}_{50}\right)$ and cytotoxities $\left(\mathrm{CC}_{50}\right)$ in cell-based assays, in addition the selectivity index (SI) was calculated (Table 1). The most active compound was (E)- $N-(2-$ bromostyryl)- $N-3$ ', 5' -difluorobenzylacetamide (5i, $\left.\mathrm{EC}_{50}=4 \mu \mathrm{m}, \mathrm{SI}=29\right)$. Compound $\mathbf{5 f}\left(\mathrm{IC}_{50}=320 \mu \mathrm{m}\right.$, $\left.\mathrm{EC}_{50}=8 \mu \mathrm{m}\right)$ is 40 times more potent in cell culture than in enzyme assays. One possibility for this discrepancy was supposed to be that the structure of compound $\mathbf{5 f}$ was different from other $N$-alkylated compounds. In compound $\mathbf{5 f}$, two acetyl groups were substituted on the nitrogen atom, and this change in structure might cause compound $\mathbf{5 f}$ active to other viral targets. Further structure modification of this kind of compounds is currently underway in our lab.

\section{Experimental}

\subsection{General}

Unless otherwise stated, all reagents were commercial analytical or chemical pure grades and were not additionally purified. IR spectra were measured on a Bio-Rad FTS-135 spectrometer (Bio-Rad, Richmond, CA) with $\mathrm{KBr}$ pellets, $v$ in $\mathrm{cm}^{-1}$; EI-MS data were recorded on an AutoSped 300 instrument; ${ }^{1} \mathrm{H}-\mathrm{NMR}$ spectra were recorded in $\mathrm{CDCl}_{3}$ on Bruker AM 400 or DRX-500 spectrometers. Chemical shifts are given in $\delta(\mathrm{ppm})$ with TMS as internal reference; column chromatography (CC): silica gel (200-300 mesh; Qingdao Marine Chemical Inc.; Qingdao; China).

\subsection{Preparation of (E)- $\beta$-phenylnitroolefins 2}

An ortho-substituted benzaldehyde $(2 \mathrm{mmol})$, ammonium acetate $(0.1 \mathrm{~g}, 2.6 \mathrm{mmol})$ and nitromethane $(3 \mathrm{~mL})$ were placed in a $10 \mathrm{~mL}$ round bottomed flask equipped with a condenser. The mixture was refluxed and stirred for 2 hours and then evaporated to give a residue that was dissolved in $\mathrm{CH}_{2} \mathrm{Cl}_{2}(5 \mathrm{~mL})$, washed successively with saturated brine and water, and the organic layer was evaporated to give the target compounds that were used without further purification (yields: 90-95\%).

\subsection{Preparation of (E)-N-phenylstyrylacetamides $\mathbf{3}$}

Under a $\mathrm{N}_{2}$ atmosphere, the appropriate compound 2 (1 mmol), iron powder $(1.12 \mathrm{~g}, 20 \mathrm{mmol})$, acetic acid $(0.2 \mathrm{~mL})$ and acetic anhydride $(6 \mathrm{~mL})$ were added to a $20 \mathrm{~mL}$ round flask with a condenser. The mixture was refluxed for 2 hours and then cooled to room temperature. Methanol (20 mL) was 
added to dilute the mixture, which was filtered through Celite ${ }^{\circledR}$. The filtrate was poured onto ice cooled water $(50 \mathrm{~mL})$ and then extracted with ethyl acetate. The organic layer was evaporated to a residue and dissolved in methanol $(20 \mathrm{~mL})$. The methanolic solution was adjusted to $\mathrm{pH} 12-14$ with methanolic potassium hydroxide to hydrolyze diacetyl compounds $\mathbf{4}$ to target compounds $\mathbf{3}$ and this basic solution was poured onto water $(50 \mathrm{~mL})$ and extracted again with ethyl acetate. The organic layer was washed successively with saturated brine and water, dried over anhydrous $\mathrm{Na}_{2} \mathrm{SO}_{4}$, and then evaporated to give a crude product which was purified on a silica gel column eluted with hexane/ethyl acetate $(4: 1, \mathrm{~V} / \mathrm{V})$ to give compounds 3 (yields: $45-50 \%)$.

3.4. General procedure for the preparation of (E)- $N$-phenylstyryl- $N$-alkylacetamides 5: Preparation of (E)-N-(3-bromostyryl)-N-methylacetamide (5a)

A solution of compound $3\left(\mathrm{R}^{1}=3-\mathrm{Br}, 98 \mathrm{mg}, 0.5 \mathrm{mmol}\right)$ in dry tetrahydrofuran $(5 \mathrm{~mL})$ was cooled to $0{ }^{\circ} \mathrm{C}$, sodium hydride $(0.6 \mathrm{mmol})$ was added, the mixture was kept stirring at $0^{\circ} \mathrm{C}$ for $30 \mathrm{~min}$, then a solution of iodomethane $(0.6 \mathrm{mmol})$ in tetrahydrofuran $(1 \mathrm{~mL})$ was added. The mixture was stirred at $0^{\circ} \mathrm{C}$ for another $30 \mathrm{~min}$, then warmed up to ambient temperature and stirred until the completion of the reaction as monitored by TLC. The mixture was poured onto ice cooled water and extracted with ethyl acetate, the organic layer was then washed with brine and dried with anhydrous $\mathrm{Na}_{2} \mathrm{SO}_{4}$. After evaporation, the residue was purified on silica gel chromatography giving the target compound 5a as a white powder; yield: $53 \%$; ${ }^{1} \mathrm{H}-\mathrm{NMR}\left(\mathrm{CDCl}_{3}\right) \delta: 8.11(\mathrm{~d}, J=14.9 \mathrm{~Hz}, 1 \mathrm{H}), 7.53-7.15(\mathrm{~m}, 4 \mathrm{H}), 5.90(\mathrm{~d}$, $J=14.9 \mathrm{~Hz}, 1 \mathrm{H}), 3.25(\mathrm{~s}, 3 \mathrm{H}), 2.36(\mathrm{~s}, 3 \mathrm{H})$; IR $v_{\max }(\mathrm{KBr}) \mathrm{cm}^{-1}: 1670,1634$; EI-MS m/z (\%): 255 $[\mathrm{M}+2]^{+}(75), 253[\mathrm{M}]^{+}$(79), 213 (95), 211 (100), 185 (13), 183(13), 131 (15). Compounds 5b-5t (structures provided in Table 1) were synthesized using the above method.

(E)-N-(4-bromostyryl)-N-methylacetamide (5b): White powder, yield: 69\%; ${ }^{1} \mathrm{H}-\mathrm{NMR} \delta: 8.13$ (d, $J=14.9 \mathrm{~Hz}, 1 \mathrm{H}), 7.43(\mathrm{~d}, J=8.2 \mathrm{~Hz}, 2 \mathrm{H}), 7.20$ (d, $J=8.2 \mathrm{~Hz}, 2 \mathrm{H}), 5.92(\mathrm{~d}, J=14.9 \mathrm{~Hz}, 1 \mathrm{H}), 3.26$ (s, $3 \mathrm{H}), 2.36(\mathrm{~s}, 3 \mathrm{H})$; IR $v_{\max } \mathrm{cm}^{-1}: 1674,1638$; EI-MS m/z (\%): $255[\mathrm{M}+2]^{+}$(40), $253[\mathrm{M}]^{+}(43), 213$ (90), 211 (100), 185 (10), 183(10), 168 (33), 149 (37), 131 (30), 117 (20).

(E)- $N$-(2-bromostyryl)- $N$-methylacetamide (5c): White powder, yield $50 \% ;{ }^{1} \mathrm{H}-\mathrm{NMR} \delta: 8.00$ (d, $J=15.0 \mathrm{~Hz}, 1 \mathrm{H}$ ), 7.55-7.00 (m, 4H), $6.22(\mathrm{~d}, J=15.0 \mathrm{~Hz}, 1 \mathrm{H}), 3.24(\mathrm{~s}, 3 \mathrm{H}), 2.28(\mathrm{~s}, 3 \mathrm{H}) ; \mathrm{IR} v_{\max } \mathrm{cm}^{-1}$ : 1680, 1632; EI-MS m/z (\%): $255[\mathrm{M}+2]^{+}$(25), $253[\mathrm{M}]^{+}, 213$ (50), 211 (50), 185 (55), 183(55), 132 (100), 117 (67).

(E)-N-(2-bromostyryl)-N-ethylacetamide (5d): White powder, yield 65\%; ${ }^{1} \mathrm{H}-\mathrm{NMR} \delta: 7.85$ (d, $J=14.6 \mathrm{~Hz}, 1 \mathrm{H}), 7.54-7.02(\mathrm{~m}, 4 \mathrm{H}), 6.27$ (d, $J=14.6 \mathrm{~Hz}, 1 \mathrm{H}), 3.82(\mathrm{q}, J=7.0 \mathrm{~Hz}, 2 \mathrm{H}), 2.28(\mathrm{~s}, 3 \mathrm{H})$, $1.31(\mathrm{t}, J=7.0 \mathrm{~Hz}, 3 \mathrm{H})$; IR $v_{\max } \mathrm{cm}^{-1}: 1677,1632,1399,1246$; EI-MS m/z (\%): $268[\mathrm{M}+2]^{+}$(45), 267 [M] (45), 227 (75), 225 (76), 210 (38), 188 (32), 130 (100).

(E)-N-(2-bromostyryl)- $N$-allylacetamide (5e): Viscous oil, yield 65\%; ${ }^{1} \mathrm{H}-\mathrm{NMR} \delta: 7.95$ (d, J=14.2 Hz, $1 \mathrm{H}), 7.55-7.04(\mathrm{~m}, 4 \mathrm{H}), 6.33(\mathrm{~d}, J=14.2 \mathrm{~Hz}, 1 \mathrm{H}), 5.86-5.83(\mathrm{~m}, 1 \mathrm{H}), 5.31-5.02(\mathrm{~m}, 2 \mathrm{H}), 4.43-4.29(\mathrm{~m}$, 2H), 2.24 (s, 3H); IR $v_{\max } \mathrm{cm}^{-1}: 1678,1632,1395,1225$; EI-MS m/z (\%): $281[\mathrm{M}+2]^{+}(90), 279$ [M] (100), 239 (87), 237 (94), 224 (40), 222 (48), 158 (80), 156 (37), 143 (27), 130 (51), 117 (83). 
(E)- $N$-(2-bromostyryl)-N-acetylacetamide (5f): White powder, yield 55\%; ${ }^{1} \mathrm{H}-\mathrm{NMR} \delta: 7.56$ (d, $J=7.9 \mathrm{~Hz}, 2 \mathrm{H}), 7.30(\mathrm{t}, J=7.4 \mathrm{~Hz}, 1 \mathrm{H}), 7.16(\mathrm{t}, \mathrm{J}=7.7 \mathrm{~Hz}),, 6.85(\mathrm{~d}, J=14.4 \mathrm{~Hz}, 1 \mathrm{H}), 6.76(\mathrm{~d}$, $J=14.4 \mathrm{~Hz}, 1 \mathrm{H}), 2.44(\mathrm{~s}, 6 \mathrm{H})$; IR $v_{\max } \mathrm{cm}^{-1}: 1715,1644,1468,1426,1368,1274,1228 ;$ FAB-MS $\mathrm{m} / \mathrm{z}$ (\%): $282[\mathrm{M}+1]^{+}(100)$.

(E)-N-(2-bromostyryl)- $N$-benzylacetamide (5g): White powder, yield $67 \%$; mp $86-88{ }^{\circ} \mathrm{C} ;{ }^{1} \mathrm{H}-\mathrm{NMR} \delta$ : $8.08(\mathrm{~d}, J=14.3 \mathrm{~Hz}, 1 \mathrm{H}), 7.48-7.02(\mathrm{~m}, 9 \mathrm{H}), 6.30-6.19(\mathrm{~m}, 1 \mathrm{H}), 5.03,(\mathrm{~s}, 2 \mathrm{H}), 2.43(\mathrm{~s}, 3 \mathrm{H})$; IR $v_{\max }$ $\mathrm{cm}^{-1}$ : IR $v_{\max } \mathrm{cm}^{-1}: 1663,1578,1398,1322,1256,1229$; EI-MS m/z (\%): $331[\mathrm{M}+2]^{+}$(20), 329 [M] (22), 289 (23), 287 (25), 250 (8), 208 (10), 91 (100).

(E)-N-(2-bromostyryl)-N-2',6'-difluorobenzylacetamide (5h): White powder, yield 70\%; ${ }^{1} \mathrm{H}-\mathrm{NMR} \delta$ : 7.50-6.80 (m, 8H), $6.27(\mathrm{~d}, J=14.2 \mathrm{~Hz}, 1 \mathrm{H}), 5.20(\mathrm{~s}, 2 \mathrm{H}), 2.38(\mathrm{~s}, 3 \mathrm{H})$; IR $v_{\max } \mathrm{cm}^{-1}$ : 1669, 1627, 1469, 1406, 1316. EI-MS m/z (\%): $367[\mathrm{M}+2]^{+}(15), 365[\mathrm{M}]^{+}$(15), 325 (35), 323 (27), 286 (7), 244 (15), 127 (100).

(E)-N-(2-bromostyryl)- $N$-3',5'-difluorobenzylacetamide (5i): White powder, yield $66 \%$; ${ }^{1} \mathrm{H}-\mathrm{NMR} \delta$ : 7.52-6.67 (m, 8H), $6.17(\mathrm{~d}, J=14.5 \mathrm{~Hz}, 1 \mathrm{H}), 5.00(\mathrm{~s}, 2 \mathrm{H}), 2.43(\mathrm{~s}, 3 \mathrm{H})$; IR $v_{\max } \mathrm{cm}^{-1}: 160,1635,1598$, 1461, 1437, 1396, 1319; EI-MS m/z (\%): $367[\mathrm{M}+2]^{+}$(45), $365[\mathrm{M}]^{+}$(48), 325 (83), 323 (90), 286 (20), 244 (57), 127 (100). 117 (95).

(E)-N-(2-bromostyryl)- $N$-2',6'-dichlorobenzylacetamide (5j): White powder, yield $67 \%$; ${ }^{1} \mathrm{H}-\mathrm{NMR} \delta$ : 7.53-6.94 (m, 8H), $6.21(\mathrm{~d}, \mathrm{~J}=12.9 \mathrm{~Hz}, 1 \mathrm{H}), 5.35(\mathrm{~s}, 2 \mathrm{H}), 2.35(\mathrm{~s}, 3 \mathrm{H}) ; \mathrm{IR} v_{\max } \mathrm{cm}^{-1}: 1674,128,1580$, 1435, 1402, 1340; EI-MS m/z (\%): $401[\mathrm{M}+4]^{+}(10), 399[\mathrm{M}+2]^{+}(20), 397[\mathrm{M}]^{+}$(15), 357 (40), 322 (64), 320 (58), 161 (68), 159 (100) 117 (42).

(E)-N-(2-chlorostyryl)- $N$-methylacetamide (5k): White powder yield: $67 \% ;{ }^{1} \mathrm{H}$ NMR $\delta: 8.07$ (d, $J=14.9 \mathrm{~Hz}, 1 \mathrm{H}), 7.43-7.13(\mathrm{~m}, 4 \mathrm{H}), 6.27(\mathrm{~d}, J=14.9 \mathrm{~Hz}), 3.26(\mathrm{~s}, 3 \mathrm{H}) ; 2.30(\mathrm{~s}, 3 \mathrm{H}) ; \mathrm{IR} v_{\max } \mathrm{cm}^{-1}$ : 1680, 1632, 1387, 1332, 1256; EI-MS m/z (\%): $211[\mathrm{M}+2]^{+}(25), 209[\mathrm{M}]^{+}$(75), 169 (32), 167 (100), $132(100), 130(35)$.

(E)-N-(2-chlorostyryl)-N-ethylacetamide (5l): White powder, yield: 73\%; ${ }^{1} \mathrm{H}-\mathrm{NMR} \delta: 7.89$ (d, $J=14.6 \mathrm{~Hz}, 1 \mathrm{H}), 7.39-7.07(\mathrm{~m}, 4 \mathrm{H}), 6.29$ (d, $J=14.6 \mathrm{~Hz}, 1 \mathrm{H}), 3.81(\mathrm{q}, J=6.9 \mathrm{~Hz}, 2 \mathrm{H}), 2.27(\mathrm{~s}, 2 \mathrm{H})$, $1.29(\mathrm{t}, \mathrm{J}=6.9 \mathrm{~Hz}, 3 \mathrm{H})$; IR $v_{\max } \mathrm{cm}^{-1}: 1677,1633,1440,1399,1246$; EI-MS m/z (\%): $225[\mathrm{M}+2]^{+}(28)$, $223[\mathrm{M}]^{+}(85), 183(33), 181$ (100), 168 (24), 166 (68), 146 (32), 130 (64).

(E)-N-(2-chlorostyryl)-N-allylacetamide (5m): White powder, yield: 63\%; ${ }^{1} \mathrm{H}-\mathrm{NMR} \delta: 8.00$ (d, $J=14.5 \mathrm{~Hz}, 1 \mathrm{H}), 7.51-7.07(\mathrm{~m}, 4 \mathrm{H}), 6.34-6.26(\mathrm{~m}, 1 \mathrm{H}), 5.91-5.60(\mathrm{~m}, 1 \mathrm{H}), 5.28-5.19(\mathrm{~m}, 2 \mathrm{H}), 4.26(\mathrm{~d}$, $J=5.0 \mathrm{~Hz}, 2 \mathrm{H}), 2.32$ (s, 3H); IR $v_{\max } \mathrm{cm}^{-1}: 1674,1632,1440,1397$; EI-MS m/z (\%): $237[\mathrm{M}+2]^{+}$(5), $235[\mathrm{M}]^{+}(14), 193(10), 178(12), 141(100), 139$ ( 87), 77 (100). 
(E)-N-(2-fluorostyryl)-N-methylacetamide (5n): White powder; yield: 70\%; ${ }^{1} \mathrm{H}-\mathrm{NMR} \delta: 8.12$ (d, $J=15.0 \mathrm{~Hz}, 1 \mathrm{H}), 7.51-7.03(\mathrm{~m}, 4 \mathrm{H}), 6.07(\mathrm{~d}, J=15.0 \mathrm{~Hz}, 1 \mathrm{H}), 3.23(\mathrm{~s}, 3 \mathrm{H}) ; 2.30(\mathrm{~s}, 3 \mathrm{H}) ; \mathrm{IR} v_{\max } \mathrm{cm}^{-1}$ : 1671, 1637, 1488, 1457, 1385, 1314; EI-MS m/z (\%): 193 [M] $]^{+}$(60), 151 (100), 109(50).

(E)- $N$-(2-fluorostyryl)- $N$-allylacetamide (5o): White powder, yield: $63 \%$; ${ }^{1} \mathrm{H}-\mathrm{NMR} \quad \delta: 8.10$ (d, $J=14.7 \mathrm{~Hz}, 1 \mathrm{H}), 7.44-7.01(\mathrm{~m}, 4 \mathrm{H}), 6.07-6.00(\mathrm{~m}, 1 \mathrm{H}), 5.90-5.81(\mathrm{~m}, 1 \mathrm{H}), 5.28-5.04(\mathrm{~m}, 2 \mathrm{H}), 4.27$ (d, $J=5.0 \mathrm{~Hz}, 2 \mathrm{H}), 2.34$ (s, 3H); IR $v_{\max } \mathrm{cm}^{-1}: 1679,1636,1490,1395$; EI-MS m/z (\%): 219 [M] (78), 177 (67), 162 (57), 148 ( 28), 135 (35), 109 (100), 101 (26), 68 (72).

(E)-N-(2-methylstyryl)-N-methylacetamide (5p): White powder, yield: 65\%; ${ }^{1} \mathrm{H}-\mathrm{NMR} \delta$ : 7.97 (d, $J=15.0 \mathrm{~Hz}, 1 \mathrm{H}), 7.45-7.12(\mathrm{~m}, 4 \mathrm{H}), 6.09$ (d, $J=15.0 \mathrm{~Hz}), 3.24(\mathrm{~s}, 3 \mathrm{H}) ; 2.35$ (s, 3H); 2.29 (s, 3H); IR $v_{\max } \mathrm{cm}^{-1}: 1668,1633,1468,1440,1382,1310$, ; EI-MS m/z (\%): $189\left[\mathrm{M}^{+}(67), 147\right.$ (100), 132 (20), 106 (30). 73 (57).

(E)-N-(2-methylstyryl)-N-allylacetamide (5q): White powder, yield: 61\%; ${ }^{1} \mathrm{H}-\mathrm{NMR} \quad \delta$ : 7.90 (d, $J=14.7 \mathrm{~Hz}, 1 \mathrm{H}), 7.45-7.07(\mathrm{~m}, 4 \mathrm{H}), 6.18-6.11(\mathrm{~m}, 1 \mathrm{H}), 5.87-5.84(\mathrm{~m}, 1 \mathrm{H}), 5.28-5.18(\mathrm{~m}, 2 \mathrm{H}), 4.44(\mathrm{~d}$, $J=5.0 \mathrm{~Hz}, 2 \mathrm{H}), 2.33(\mathrm{~s}, 3 \mathrm{H}), 2.30(\mathrm{~s}, 3 \mathrm{H})$; IR $v_{\max } \mathrm{cm}^{-1}: 1675,1635,1396$; EI-MS m/z (\%): 215 [M] $]^{+}$ (100), 173 (42), 158 (53), 130 (44), 117 (46), 105 (41) 68 (42).

(E)-N-(2,4-dimethoxystyryl)- $N$-methylacetamide (5r): White powder, yield: 73\%; ${ }^{1} \mathrm{H}-\mathrm{NMR} \delta$ : $7.90(\mathrm{~d}$, $J=15.0 \mathrm{~Hz}, 1 \mathrm{H}), 7.39-7.18(\mathrm{~m}, 2 \mathrm{H}), 6.47(\mathrm{~s}, 1 \mathrm{H}), 6.11(\mathrm{~d}, J=15.0 \mathrm{~Hz}, 1 \mathrm{H}), 3.85,3.81$ (s, each $3 \mathrm{H})$, $3.21(\mathrm{~s}, 3 \mathrm{H}), 2.28(\mathrm{~s}, 3 \mathrm{H})$; IR $v_{\max } \mathrm{cm}^{-1}: 1672,1634,1580,1508,1461,1416,1390,1332$, 1288; EI-MS m/z (\%): $235[\mathrm{M}]^{+}$(100), 193 (20), 192 (20), 178 (30), 161 (30), 137 (22).

(E)-N-(2,4-dimethoxystyryl)-N-ethylacetamide (5s): White powder, yield: 69\%; ${ }^{1} \mathrm{H}-\mathrm{NMR} \delta: 7.85(\mathrm{~d}$, $J=14.7 \mathrm{~Hz}, 1 \mathrm{H}), 7.23-7.17$ (m, 2H), $6.46(\mathrm{~s}, 1 \mathrm{H}), 6.15$ (d, $J=14.7 \mathrm{~Hz}, 1 \mathrm{H}), 3.83,3.80$ (s, each 3H), $3.68(\mathrm{q}, J=6.7 \mathrm{~Hz}, 2 \mathrm{H}), 2.24(\mathrm{~s}, 3 \mathrm{H}), 1.19(\mathrm{t}, \mathrm{J}=6.7 \mathrm{~Hz}, 3 \mathrm{H}) ; \mathrm{IR} v_{\max } \mathrm{cm}^{-1}: 1668,1633,1608,1579$, 1505, 1462, 1402; EI-MS m/z (\%): 249 [M] ${ }^{+}$(100), 207 (15), 206 (19), 175 (17), 160 (13), 151 (15), $70(57)$.

(E)-N-(2,4-dimethoxystyryl)-N-allylacetamide (5t): White powder, yield: 65\%; ${ }^{1} \mathrm{H}-\mathrm{NMR} \delta: 7.94(\mathrm{~d}$, $J=15.1 \mathrm{~Hz}, 1 \mathrm{H}), 7.34-7.15(\mathrm{~m}, 2 \mathrm{H}), 6.45(\mathrm{~s}, 1 \mathrm{H}), 6.15(\mathrm{~d}, J=15.1 \mathrm{~Hz}, 1 \mathrm{H}), 5.87-5.80$ (m, 1H), 5.25$5.10(\mathrm{~m}, 2 \mathrm{H}), 3.39(\mathrm{~d}, J=4 \mathrm{~Hz}, 2 \mathrm{H}), 3.83,3.73(\mathrm{~s}$, each $3 \mathrm{H}), 2.29(\mathrm{~s}, 3 \mathrm{H}) ; \mathrm{IR} v_{\max } \mathrm{cm}^{-1}: 1671,1634$, 160, 1579, 1505, 1398; EI-MS m/z (\%): $261[\mathrm{M}]^{+}$(92), 221 (45), 219 (53), 177 (42), 162 (41), 147 (81), 109 (42), 73 (100).

\subsection{Biological activity assays}

Compounds 5 were assayed for their anti-HIV-1 activities as reported previously [14-16].

HIV-1 reverse transcriptase assay: The inhibition of compounds 5 on recombinant HIV-1 RT activity was determined with a commercially available ELISA kit (Roche Molecular Biochemicals). The compounds were incubated with DIG-labeled-reaction mixture at $37{ }^{\circ} \mathrm{C}$ for $15 \mathrm{~h}$, and then anti-DIG- 
POD solution was added, followed by substrate ABTS. Foscarnet was used as a positive compound. The absorbance at $405 \mathrm{~nm} / 490 \mathrm{~nm}$ (A405/490) was read in the ELISA reader mentioned above. Inhibition ratio on recombinant RT activity was determined from the dose-response curve.

Cell based anti-HIV-1 bioassay: C8166 cells were maintained in RPMI-1640 supplemented with 10\% heat inactivated newborn calf serum (Gibco), The cells used in all experiments were in log-phase growth. 3'-Azido-3'-deoxythymidine (AZT), the positive control, was purchased from Sigma (USA).

Syncytium reduction assay: In the presence of $100 \mu \mathrm{L}$ of various concentrations of compounds, C8166 cells $\left(4 \times 10^{5} \mathrm{~mL}^{-1}\right)$ were infected with HIV-1 ${ }_{\text {IIIB }}$ at a multiplicity of infection (MOI) of 0.06 . The final volume per well was $200 \mu \mathrm{L}$. AZT was used as a positive control. After 3 days of culture, the cytopathic effect (CPE) was measured by counting the number of syncytia (multinucleated giant cell) in each well under an inverted microscope. Percentage inhibition of syncytial cell number in treated culture to that in infected control culture and $50 \%$ effective concentration $\left(\mathrm{EC}_{50}\right)$ was calculated.

Cytotoxicity assay: The cellular toxicity of compounds on C8166 cells was assessed by MTT methods. Briefly, cells were seeded on a microplate in the absence or presence of various concentrations of compounds in triplicate and incubated at $37{ }^{\circ} \mathrm{C}$ in a humid atmosphere of $5 \% \mathrm{CO}_{2}$ for $72 \mathrm{~h}$. The supernatants were discarded and MTT reagent $(5 \mathrm{mg} / \mathrm{mL}$ in PBS) was added to each wells, then incubated for $4 \mathrm{~h}, 100 \mu \mathrm{L}$ of $50 \%$ DMF-20\% SDS was added. After the formazan was dissolved completely, the plates were read on a Bio-Tek ELx 800 ELISA reader at 595/630 nm. The cytotoxic concentration that caused the reduction of viable cells by $50 \%\left(\mathrm{CC}_{50}\right)$ was calculated from doseresponse curve.

\section{Conclusions}

We have synthesized a series of (E)-N-phenylstyryl- $N$-alkylacetamides. Evaluation of the title compounds as NNRTIs of HIV-1 in vitro showed that compound $\mathbf{5} \mathbf{c}$ showed a higher TI value than its isomer 5a, which suggested that higher enzymatic inhibitory ratio would lead to increased anti-HIV activity in cell-based assay. Compound $\mathbf{5 i}$ exhibited the highest inhibition activity in both enzyme and cell based assays. Although the TI values of the tested compounds were suboptimal, the title compounds possessed low molecular weight and contained a naturally occurring structure unit (hamigeroxalamic acid). The SAR exploration provided us a guide for further anti-HIV-1 study of the derivatives in this family. Compound $5 \mathbf{i}$ represented a new lead for the design and synthesis of more potent and selective analogues act as NNRTIs. Further structure modification and anti-HIV-1 activities of compounds 5 are underway.

\section{Acknowledgements}

This work was partly supported by young teachers' scientific researching foundation for Dr Pi Cheng in Centre South University. 


\section{References and Notes}

1. Schinazi, R.F.; Hernandez-Santiago, B.I.; Hurwitz, S.J. Pharmacology of current and promising nucleosides for the treatment of human immunodeficiency viruses. Antivir. Res. 2006, 71, $322-334$.

2. Didierjean, J.; Isel, C.; Querré, F.; Mouscadet, J.F.; Aubertin, A.M.; Valnot, J.Y.; Piettre, S.R.; Marquet, R. Inhibition of human immunodeficiency virus type 1 reverse transcriptase, RNase H, and integrase activities by hydroxytropolones. Antimicrob. Agents Chemother. 2005, 49, 4884-4894.

3. Yeni, P. Update on HAART in HIV. J. Hepatol. 2006, 44, S100-S103.

4. Xu, Y.S.; Zeng, C.C.; Jiao, Z.G.; Hu, L.M.; Zhong, R.G. Design, synthesis and anti-HIV integrase evaluation of 4-oxo-4H-quinolizine-3-carboxylic acid derivatives. Molecules 2009, 14, 868-883.

5. Jones, L.H.; Allan, G.; Barba, O.; Burt, C.; Corbau, R.; Dupont, T.; Knochel, T.; Irving, S.; Middleton, D.S.; Mowbray, C.E.; Perros, M.; Ringrose, H.; Swain, N.A.; Webster, R. Westby, M. Phillips, O. Novel indazole non-nucleoside reverse transcriptase inhibitors using molecular hybridization based on crystallographic overlay. J. Med. Chem. 2009, 52, 1219-1223.

6. Wu, J.; Liu, X.; Cheng, X.; Cao, Y.; Wang, D.; Li, Z.; Xu, W.; Pannecouque, C.; Witvrouw, M.; Clercq, E.D. Synthesis of novel derivatives of 4-amino-3-(2-furyl)-5-mercapto-1,2,4-triazole as potential HIV-1 NNRTIs. Molecules 2007, 12, 2003-2016.

7. Barreca, M.L.; Rao, A.; Luca, L.D.; Iraci, N.; Monforte, A.M.; Maga, G.; Clercq, E.D.; Pannecouque, C.; Balzarini, J.; Chimirri, A. Discovery of novel benzimidazolones as potent nonnucleoside reverse transcriptase inhibitors active against wild-type and mutant HIV-1 strains. Bioorg. Med. Chem. Lett. 2007, 17, 1956-1960.

8. Gagnon, A.; Landry, S.; Coulombe, R.; Jakalian, A.; Guse, I.; Thavonekham, B.; Bonnear, P.R.; Yoakim, C.; Simoneau, B. Investigation on the role of the tetrazole in the binding of thiotetrazolylacetanilides with HIV-1 wild type and K103N/Y181C double mutant reverse transcriptases. Bioorg. Med. Chem. Lett. 2009, 19, 1199-1205.

9. Billamboz, M.; Bailly, F.; Barreca, M.L.; Luca, L.D.; Mouscadet, J.; Calmels, C.; Andréola, M.; Witvrouw, M.; Christ, F.; Debyser, Z.; Cotelle, P. Design, synthesis, and biological evaluation of a series of 2-hydroxyisoquinoline-1,3(2H,4H)-diones as dual inhibitors of human immunodeficiency virus type 1 integrase and the reverse transcriptase RNase H domain. J. Med. Chem. 2008, 51, 7717-7730.

10. Burke, J.T.R.; Fesen, M.R.; Mzaumder, A.; Wang, J.; Carothers, A.M.; Grunberger, D.; Driscoll, J.; Kohn, K.; Pormier, Y. Hydroxylated aromatic inhibitors of HIV-1 integrase. J. Med. Chem. 1995, 38, 4171-4178.

11. Cheng, P.; Jiang, Z.Y.; Wang, R.R.; Zhang, X.M.; Wang, Q.; Zheng, Y.T.; Zhou, J.; Chen, J.J. Synthesis and biological evaluation of $\mathrm{N}$-acetyl-beta-aryl-1,2-didehydroethylamines as new HIV1 RT inhibitors in vitro. Bioorg. Med. Chem. Lett. 2007, 17, 4476-4480.

12. Cheng, P.; Huang, N.; Jiang, Z.Y.; Zhang, Q.; Zheng, Y.T.; Chen, J.J.; Zhang, X.M.; Ma, Y.B. 1Aryltetrahydroisoquinolines as active anti-HIV agents in vitro. Bioorg. Med. Chem. Lett. 2008, 18, 2475-2478.

13. Yan, M.H.; Cheng, P.; Jiang, Z.Y.; Ma, Y.B.; Zhang, X.M.; Zhang, F.X.; Yang, L.M.; Zheng, 
Y.T.; Chen, J.J. Periglaucines A-D, anti-HBV and HIV-1 alkaloids from Pericampylus glaucus. J. Nat. Prod. 2008, 71, 760-763.

14. Wang, Q.; Wang, Y.Y.; Pu, S.P.; Zheng, Y.T. Zinc coupling potentiates anti-HIV-1 activity of baicalin. Biochem. Biophys. Res. Commun. 2004, 324, 605-610.

15. Jiang, T.; Kuhen, K.L.; Wolff, K.; Yin, H.; Bieza, K.; Caldwell, J.; Bursulaya, B.; Wu, T.Y.; He, Y. Design, synthesis and biological evaluations of novel oxindoles as HIV-1 non-nucleoside reverse transcriptase inhibitors. Part I. Bioorg. Med. Chem. Lett. 2006, 16, 2105-2108.

16. Barreca, M.L.; Rao, A.; Laura, D.L.; Zappalà, M.; Monforte, A.; Maga, G.; Pannecouque, C.; Balzarini, J.; Clercq, E.D.; Chimirri, A.; Monforte, P. Computational strategies in discovering novel non-nucleoside inhibitors of HIV-1 RT. J. Med. Chem. 2005, 48, 3433-3437.

Sample Availability: Samples of the compounds 5a-5t are available from the authors.

(C) 2009 by the authors; licensee Molecular Diversity Preservation International, Basel, Switzerland. This article is an open-access article distributed under the terms and conditions of the Creative Commons Attribution license (http://creativecommons.org/licenses/by/3.0/). 